\title{
Tisk stom the
}

\section{Évaluation de l'impact d'un jeu sérieux en réalité mixte}

\author{
Sébastien GEORGE (LIUM, Université du Maine), \\ Christine MICHEL, Audrey SERNA (LIRIS, INSA-Lyon), \\ Luca BISOGNIN (Société Symetrix)
}

RÉSUMÉ • Les jeux sérieux en réalité mixte sont des environnements qui combinent les jeux à buts éducatifs et des technologies de réalité mixte. Ces environnements peuvent fournir de nouvelles opportunités en termes d'apprentissage. Néanmoins, les impacts sur l'apprentissage de tels dispositifs sont encore peu connus. Nous présentons dans cet article une étude expérimentale qui vise à évaluer ces impacts. Nous avons conçu et développé un jeu, nommé Lea(r)nIT, qui a pour but de former aux principes du Lean Management dans une école d'ingénieur. La situation d'apprentissage comporte quatre tables interactives, simulant des postes de production, sur lesquels des actions sont réalisées avec des interfaces tangibles. Nous présentons les résultats d'une étude comparative entre une session utilisant ce dispositif et une autre utilisant une version non informatisée du même jeu sérieux. Cette comparaison montre que le jeu sérieux en réalité mixte a un impact positif sur l'apprentissage, en particulier concernant les concepts théoriques de la formation.

MOTS-CLÉS • Jeu sérieux, Réalité Mixte, Table interactive, Interface tangible, Impact sur l'apprentissage

ABSTRACT • Mixed Reality Learning Games (MRLG) are digital environments which combine game-based learning and mixed reality technologies. They can provide new opportunities for learning. Nevertheless, we still know little about the learning outcomes and the situations they favor. We present in this paper an experimental study which aims to evaluate these impacts. We designed and developed a MRLG to teach Lean Management principles in an engineering school. Four multi-touch tabletops are used, each one representing a workstation in a production line on which some actions are carried with tangible interfaces. We present the results of an experiment that compares two situations, one with the MRLG and the other with a noncomputer version of the same learning game. The comparison revealed a tendency for the MRLG situation to effectively have a positive impact on learning, particularly regarding theoretical concepts.

KEYWORDS - Game-Based Learning, Mixed Reality, Tabletops, Tangible Interface, Learning Impact 
Sébastien GEORGE, Christine MICHEL, Audrey SERNA, Luca BISOGNIN

\section{Introduction}

\subsection{Jeux sérieux pour l'apprentissage}

Ces dernières années, les serious games ont pris un essor considérable et sont utilisés pour communiquer, recruter ou former. La catégorie de serious games à laquelle nous nous intéressons est celle des jeux conçus pour soutenir l'apprentissage (Learning Games en anglais, abrégés en LG), qu'ils soient informatisés ou non. Ces dispositifs marient les dimensions "action » et « émotion » de la pédagogie en rendant attrayantes la découverte de connaissances et la construction de compétences (aspect «Serious»), par une forme, des interactions, des règles et des objectifs ludiques (aspect "Games »). Les LG ont l'avantage d'offrir des environnements permettant la simulation de situations et de contextes dans lesquels les apprenants peuvent développer des aptitudes (De Freitas \& Neumann, 2009). Les simulateurs ont déjà montré des résultats probants pour l'apprentissage, notamment dans le cadre de formations professionnelles (Joab \& Gouardères, 2000). Mais les LG ne sont pas que de simples simulateurs, ils peuvent aussi immerger les apprenants dans des mondes dans lesquels ils doivent s'investir pour progresser, relever des défis et accomplir des missions. Sur certains points, les jeux sérieux peuvent s'apparenter à des simulateurs. En effet, les apprenants vont agir dans un environnement en fonction d'évènements qui se produisent et leurs actions vont avoir des conséquences. Cependant, une simulation intègre forcément un modèle d'une certaine réalité (avec un réalisme qui peut être plus ou moins poussé) : simulation de conduite d'un véhicule, simulateur de vol, simulation de circuits électroniques... Dans les cas des jeux sérieux, il n’y a pas forcément de modèle pour reproduire les situations de manière réaliste, le jeu reposant davantage sur une scénarisation. La situation choisie peut parfois être volontairement imagée et loin de la réalité, l'essentiel étant d'amener les apprenants à s'investir pour progresser, relever des défis et accomplir des missions.

Plus précisément, nous nous intéressons aux LG de type jeux de rôles, l'apprenant devant dans ce cas agir en collaboration avec d'autres pour résoudre un problème ou remplir une mission. Les apprenants peuvent ainsi vivre une situation qui serait difficile à reproduire réellement pour les raisons de coût, de temps ou de sécurité (Squire \& Jenkins, 2003). Dans son livre, Abt (1987) résume parfaitement les intérêts des jeux de rôles : "The players assume realistic roles, face problems, formulate strategies, make decisions and get fast feedback on the consequences of their action». Les LG s'appuyant sur des jeux de rôles se positionnent clairement dans les cou- 
rants de l'apprentissage contextualisé et situé (Lave \& Wenger, 1991). Pour ces chercheurs, l'apprentissage dépend de l'activité du contexte et de la culture dans lesquels il se déroule. Dans l'idéal, la connaissance doit être présentée dans des contextes authentiques (Herrington \& Oliver, 2000), c'est à dire dans des situations qui exigent normalement cette connaissance.

Les Learning Games souffrent néanmoins de certaines limites: l'artificialité de la situation d'apprentissage entraîne parfois des difficultés pour les apprenants à transférer les connaissances apprises dans d'autres situations. Ce caractère artificiel se trouve aussi bien dans les LG informatisés, de part la virtualité de l'environnement et des actions, que dans les LG non informatisés de par la manipulation d'objets souvent peu réalistes par rapport à l'activité simulée. Il a déjà été montré qu'un tuteur humain pouvait pallier ce problème et aider à expliciter les relations entre le jeu et le monde réel. Ainsi, l'«experiential learning» développée par Garris, Ahlers et Driskell (2002) met en avant la nécessité du tutorat humain (débriefing, étayage...) pour soutenir un apprentissage effectif. C'est pourquoi dans notre approche des LG, le tutorat tient une place importante. Les tuteurs régulent l'activité d'apprentissage, ils analysent les évènements qui se déroulent dans le jeu, ils illustrent les concepts dans d'autres situations, etc. L'apprentissage par l'action doit être encadré pour conduire à une abstraction des informations reçues.

\subsection{Jeux sérieux en réalité mixte}

Nous avons vu dans la partie précédente que les jeux sérieux peuvent être des environnements intéressants pour soutenir les apprentissages mais qu'ils ne permettent pas toujours le transfert des connaissances construites à des situations réelles. Nous avons vu que le tutorat humain pouvait être une solution pour pallier ce problème en proposant des débriefings sur les situations vécues dans le jeu et en illustrant les concepts abordés dans d'autres cas. Nous pensons qu'une autre solution peut être explorée, de façon complémentaire au tutorat. En effet, proposer des situations de jeu plus proches des situations réelles permet de notre point de vue de transférer plus facilement les apprentissages lorsque les apprenants sont en situations réelles, en particulier lorsqu'il est question de construction de compétences professionnelles (par exemple des procédures à exécuter dans des environnements complexes). Il existe des jeux d'entreprise en salle s'appuyant sur des mises en situations pour favoriser la construction de ce type de compétences. Ces jeux utilisent du matériel, comme des blocs de construction ou des LEGO, que les apprenants mani- 


\section{Sébastien GEORGE, Christine MICHEL, Audrey SERNA, Luca BISOGNIN}

pulent, comme des objets ou outils utilisés classiquement dans la situation, pour apprendre en jouant un rôle (CIPE, 2013). Ce type de formation a fait ses preuves mais le manque de réalisme des objets manipulés ne permet pas toujours de faire passer les concepts sous-jacents car ces objets demeurent trop symboliques et abstraits. Par exemple, si un LEGO représente une pièce usinée, la limite du symbolisme intervient lorsqu'il faut réaliser une action sur la pièce. En effet, il sera difficile pour les apprenants d'adopter une démarche inductive car certaines actions (une abrasion, une peinture) et certains phénomènes observés (un défaut d'aspect) ne seront à force plus identifiables ou compréhensibles en regardant les pièces du fait de l'accumulation de symboles. Pour augmenter le réalisme des objets et des actions, nous pensons qu'une solution est d'utiliser des techniques de réalité mixte.

Le terme Réalité Mixte (RM) fait référence à un continuum qui connecte le monde physique et le monde virtuel, englobant ainsi deux aspects (Milgram \& Kishino, 1994) :

- la Réalité Augmentée (RA), le monde réel étant enrichi par des informations virtuelles (comme dans les cockpits d'avions par exemple),

- la Virtualité Augmentée (VA), le monde virtuel étant alors enrichi par des objets bien réels (par exemple en utilisant des interfaces tangibles pour manipuler des objets virtuels).

Différents dispositifs peuvent être utilisés pour la RM : dispositifs mobiles (tablettes ou smartphone), lunettes see-through, table interactive avec interfaces tangibles, ... Ces dispositifs permettent de contrôler et/ou représenter des informations. Le fait d'ajouter des informations, non perceptibles naturellement, à des objets réels est d'un intérêt certain pour les situations d'apprentissage. Des études, comme celle de Stedmon et Stone (2001), montrent qu'en augmentant les artefacts physiques avec des informations associées, on facilite la compréhension de concepts techniques. Plus récemment, David et al. (2009) ont étudié comment des artefacts physiques (des machines industrielles ou des ordinateurs) pouvaient être augmentés de données numériques pour favoriser des apprentissages juste à temps. Même s'il est encore trop tôt pour tirer des conclusions sur les impacts de la RM sur l'apprentissage (Anastassova \& Burkhardt, 2008), il semble que les effets positifs attendus portent principalement sur l'apprentissage à court-terme. Afin de dépasser cette limite, nous pensons que des scénarios plus élaborés doivent être conçus. Nous faisons l'hypothèse qu'en couplant réalité mixte et jeu pour l'apprentissage, il est alors possible de combiner, d'une part, l'aspect motivant et situé de la RM 
et, d'autre part, le côté ludique et la scénarisation d'activités des LG. Cette hypothèse est le point de départ du projet SEGAREM (Serious Games and Mixed Reality) dans lequel le travail présenté dans cet article s'inscrit. SEGAREM vise à apporter des réponses sur le plan des méthodes, modèles et outils pour la production de dispositifs interactifs de formation innovants ; les jeux sérieux en réalité mixte (abrégés en MRLG pour Mixed Reality Learning Games). L'objectif principal de ce projet est de mettre au point un prototype d'environnement de conception, de production et d'exécution de MRLG.

Le travail présenté dans cet article se focalise sur l'évaluation des apports de la RM vis à vis d'une situation d'apprentissage par le jeu où des objets physiques sont utilisés pour représenter des outils ou des objets du monde réel. Notre hypothèse consiste à penser que le degré de réalisme et l'immersion rendus possible par la RM permet une meilleure compréhension des concepts de la formation.

Cet article est organisé de la façon suivante : dans la section 2, nous décrivons la problématique de recherche et la méthodologie de travail. Dans la section 3, nous présentons la conception d'un environnement MRLG particulier, nommé Lea(r)nIT, qui vise à former à la démarche Lean (Lean Management) en utilisant des tables interactives multi-touch, des interfaces tangibles et des tablettes. Dans la section 4, nous détaillons une évaluation expérimentale avec ce MRLG et présentons les résultats. L'article se termine par une discussion ainsi que par une présentation de pistes de recherche dans le domaine.

\section{Problématique et hypothèses de recherche}

Les impacts sur l'apprentissage des jeux en réalité mixte sont encore peu étudiés. Les questions de recherche sur les MRLG peuvent être abordées selon deux angles. D'un côté, certaines questions concernent l'utilisabilité et plus généralement l'étude de l'expérience utilisateur lors d'activités faisant intervenir des MRLG. D'autre part, et cet article se focalise sur ce point, la question de l'utilité peut être traitée: quels types d'apprentissage sont favorisés dans les MRLG par rapport à des situations sans réalité mixte ? En particulier, nous avons identifié trois hypothèses :

- H1 : à scénario pédagogique équivalent, l'apprentissage est meilleur si la simulation des outils et objets manipulés est réalisée en RM plutôt que de façon symbolique.

- H2 : à scénario pédagogique équivalent, l'apprentissage des concepts théoriques est meilleur si la simulation des outils et objets manipulés est réalisée en RM plutôt que de façon symbolique. 


\section{Sébastien GEORGE, Christine MICHEL, Audrey SERNA, Luca BISOGNIN}

- H3 : à scénario pédagogique équivalent, l'apprentissage des concepts pratiques est meilleur si la simulation des outils et objets manipulés est réalisée en RM plutôt que de façon symbolique.

Pour l'hypothèse $\mathrm{H} 1$, nous pensons que la formation avec un MRLG va favoriser l'apprentissage grâce à la manipulation d'objets moins symboliques, c'est à dire dont la représentation est plus réaliste. Ce n'est pas forcément une situation identique à la réalité qui est visée (ce qui serait le cas pour l'apprentissage de gestes techniques par exemple) mais le fait que les objets manipulés aient une représentation réaliste à la fois par rapport à une situation métier mais aussi au contexte de jeu qui a été imaginé pour la formation. Concrètement, dans le cas des MRLG, un objet (ex : une pièce à usiner) ou un outil (ex : un pistolet à colle) seront réellement représentés par des objets et outils ressemblants et non par des objets symboliques, comme des LEGO, qui requièrent une plus grande charge cognitive pour être associés à ce qu'ils représentent. Nous pensons ainsi que la manipulation d'artefacts réalistes va favoriser la compréhension globale des processus en jeu dans la situation simulée. Par ailleurs, la réalité mixte va accentuer le sentiment de plaisir, le dynamisme et l'immersion de l'apprenant. Il a déjà été montré que ces facteurs pouvaient créés des conditions favorables à l'apprentissage (Prince, 2004), essentiellement en jouant sur la motivation de l'apprenant. De plus, nous pensons que plus l'environnement est immersif, plus l'apprenant va se sentir impliqué dans la simulation. L'engagement est aussi un facteur important pour l'apprentissage.

Les hypothèses $\mathrm{H} 2$ et $\mathrm{H} 3$ vont permettre de préciser, dans le cas où $\mathrm{H} 1$ serait vérifiée, si les MRLG favorisent l'apprentissage de concepts théoriques, pratiques, ou les deux à la fois. Nous pensons fortement que les aspects pratiques et théoriques ne doivent pas être opposés, en particulier lorsqu'il s'agit de former à des compétences professionnelles. En formation professionnelle, l'utilisation de simulation est une approche couramment employée car les connaissances et les aptitudes ne peuvent pas être dissociées de l'activité et des actions (Kriz, 2010). Ainsi, nous croyons que pour l'hypothèse $\mathrm{H} 2$, un MRLG peut faciliter une meilleure représentation des concepts, en établissant des relations entre les concepts réels et les principes de plus haut niveau. Ces relations peuvent prendre la forme de connexions entre des objets réels et des informations virtuelles. De plus, les actions étant plus réalistes dans un environnement MRLG, l'activité réflexive des apprenants sur la situation est moins artificielle. 
Concernant l'hypothèse H3, les MRLG devraient supporter l'apprentissage de procédures et de processus (le savoir-faire) en étant plus à même de rendre compte de contraintes liées à des gestes techniques par exemple. En plaçant les apprenants dans des environnements très interactifs, l'apprentissage par l'action peut être amélioré. Suchman (1987) avance que les personnes raisonnent avec les objets de leur environnement (action située). Les MRLG devraient pouvoir favoriser ce type d'apprentissage situé et contextuel, en immergeant les apprenants dans un monde parallèle qui favorise l'expérience d'une autre réalité tout en portant une certaine forme d'authenticité.

\section{Jeu pour l'apprentissage en réalité mixte : une étude de cas avec Lea(r)nIT}

\subsection{Méthodes et outils pour la conception de MRLG}

Les MRLG s'appuient sur trois domaines complémentaires: la réalité mixte, les jeux et la pédagogie.

Dans le domaine de l'IHM, différents modèles permettent de structurer le processus de conception en réalité mixte. Par exemple, à un haut niveau d'abstraction, les modèles de tâches (Jourde et al., 2010) permettent de structurer hiérarchiquement les buts et les actions des utilisateurs sous forme d'arbres, alors qu'à un plus bas niveau d'abstraction, les modèles d'interaction permettent de décrire un système en réalité mixte, en distinguant les éléments numériques des objets réels.

Dans le domaine du jeu, on retrouve des outils de support et d'aide au développement. La phase de conception est quant à elle très peu assistée par des méthodes et modèles. Elle est surtout guidée selon les méthodes de travail propres à chaque concepteur. Cependant, certains concepteurs de jeux ont tout de même identifié les éléments principaux à prendre en compte lors de la conception d'un jeu, par exemple l'objectif et le sujet (Crawford, 1982).

Enfin, dans le domaine de l'éducation, des enseignants écrivent des scénarios pédagogiques pour décrire des unités d'apprentissage. En s'appuyant sur des théories d'ingénierie pédagogique (instructional design), des modèles et outils permettent de structurer et formaliser la description de scénarios pédagogiques. La comparaison de trois de ces modèles (IMSLD, LDL et ISIS) montre que ces derniers ne sont pas assez complets pour permettre de décrire l'ensemble des composants d'un MRLG (Orliac et al., 2011). En particulier, ils ne permettent pas de spécifier les dispositifs utilisés dans un MRLG : la distinction entre les objets tangibles et les objets 


\section{Sébastien GEORGE, Christine MICHEL, Audrey SERNA, Luca BISOGNIN}

numériques ne peut être précisée, pas plus que la description de l'interface numérique ou encore la position des objets physiques dans l'environnement.

À l'intersection des jeux et de la pédagogie, certains outils ont pour objectif d'assister la conception de LG. On retrouve par exemple EDoS (maintement appelé LEGADEE) (Masfisi-Schottman et al., 2010) ou encore ScenLRPG (Mariais et al., 2011). Là encore, ces outils ne sont pas adaptés pour décrire des interactions en RM.

En conclusion, les méthodes et outils utilisés dans les différents domaines sont assez éloignés les uns des autres. L'angle d'attaque des concepteurs est différent : gameplay et développement pour le domaine du jeu, organisation pédagogique (architecture d'apprentissage) pour le domaine de la pédagogie, et enfin description des interactions pour la réalité mixte. Chacun de ces domaines utilisent un vocabulaire qui lui est propre et que les autres concepteurs ne comprennent pas forcément. Pour ces raisons, aucun de ces outils ou méthodes ne peuvent être utilisés directement pour la conception de MRLG et c'est pour cela que nous avons adopté une approche combinant différents outils et méthodes. Nous proposons un modèle de conception de MRLG accompagné d'un outil auteur appelé MIRLEGADEE (Orliac et al., 2012).

Dans la suite de cet article, nous allons décrire la conception du prototype de MRLG basé sur un LG existant mais non informatisé. En adaptant un jeu existant, on s'assure d'une stabilité et d'une efficacité en terme d'apprentissage et de jeu. Deux types de transformations ont été opérés pour adapter le jeu :

- une informatisation du jeu,

- une augmentation de certains types d'interaction.

\subsection{Le jeu existant}

De façon à limiter l'incidence des choix de conception, en terme de pédagogie et de jeu, dans notre évaluation des impacts de la réalité mixte, nous avons décidé d'adapter un serious game déjà existant et de le transposer en RM. Les résultats obtenus avec ce jeu sont déjà connus et nous permettent d'avoir un cadre solide pour pouvoir ensuite évaluer les impacts de la RM sur l'apprentissage.

Nous avons choisi un jeu utilisé à l'INSA de Lyon au département Génie Industriel qui permet d'enseigner les concepts de la démarche Lean à des petits groupes d'étudiants : le Buckingham Lean Game. La démarche Lean est une méthode d'amélioration continue des systèmes de produc- 
tion. L'objectif est de rechercher la performance (en termes de productivité, de qualité, de délais et de coûts) en éliminant les gaspillages. Les objectifs d'apprentissage concernent à la fois des connaissances théoriques (par exemple les différentes techniques d'organisation de la production) et des compétences pratiques (par exemples l'application d'outils pour rééquilibrer la charge sur les postes de travail). Le jeu Buckingham Lean Game n'utilise aucune technologie informatique. Il permet, à l'aide de briques LEGO, de simuler un système de production fabriquant différentes gammes de produits. L'objectif est de répondre à la demande d'un client et fabriquer les objets sans aucun défaut qualité, en se plaçant dans une optique d'amélioration continue. Les joueurs doivent équilibrer la production pour pouvoir satisfaire la demande.

Concrètement, chaque joueur joue le rôle soit d'un opérateur responsable d'un poste de travail de la ligne de production (presse, assemblage, peinture, contrôle qualité, entrepôt) soit du client. Les joueurs sont alors acteurs lors de la simulation et travaillent sur un seul poste. Sur une table, simulant leur poste de travail, ils manipulent, assemblent ou vérifient les objets fabriqués en briques de LEGO. Des indications sur le mode opératoire de chaque poste sont fournies aux joueurs avant le début du jeu. La position de chaque table dans la classe décrit l'implantation initiale de la ligne de production ( $c f$. figure 1) : la distance entre les tables, la cohérence entre le positionnement de chaque poste de travail et le flux de production, ainsi que l'impossibilité de déplacer la presse à cause de son poids sont les contraintes initiales qui vont affecter l'efficacité et la vitesse de production.

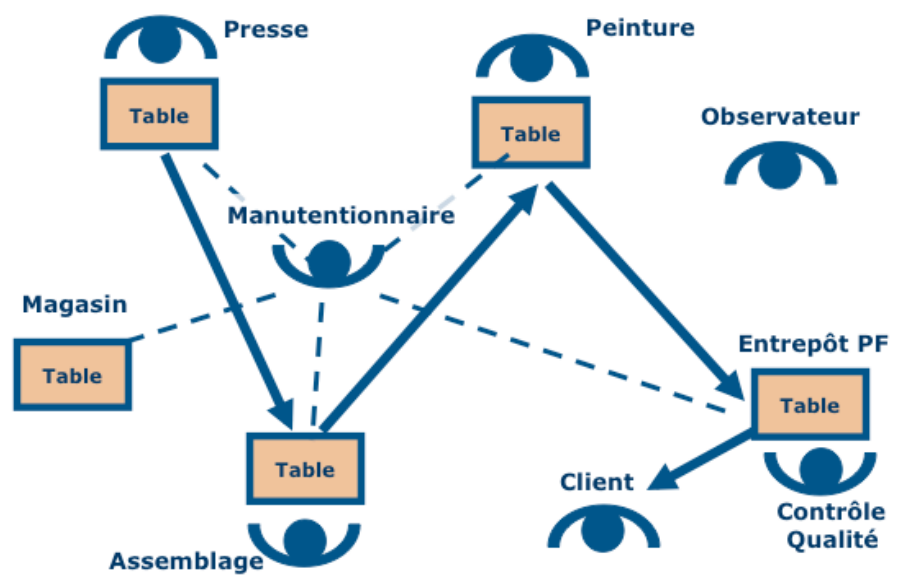

Figure 1 • Plan de la ligne de production simulée 


\section{Sébastien GEORGE, Christine MICHEL, Audrey SERNA, Luca BISOGNIN}

À partir de cette configuration de base, une première phase de simulation a lieu et la production commence. De nombreux problèmes sont révélés lors de cette première simulation, en particulier concernant l'entrepôt dans lequel il manque des produits et le manutentionnaire qui est submergé. À la fin de la simulation, l'enseignant introduit des indicateurs de Lean, utiles pour l'analyse de l'activité, tels que le taux non qualité, le taux de production, le taux d'éléments livré en juste-à-temps, les surplus de production, le temps moyen de livraison, le facteur stress, etc. Les étudiants analysent ces indicateurs avec l'aide de l'enseignant puis proposent des améliorations suivant la démarche du Lean. Ils établissent ainsi un plan d'action et l'appliquent au système de production. Pour vérifier et valider les effets de ces changements, d'autres simulations sont réalisées ( $c f$. figure 2 ).

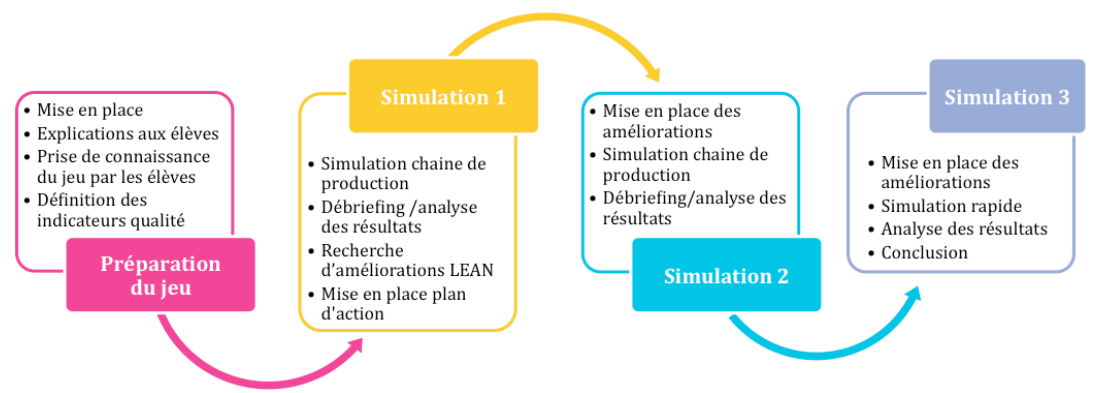

Figure 2 • Les différentes étapes du jeu Buckingham Lean

Avec ce fonctionnement, les étudiants alternent entre observation, planification et action. De plus, le scénario pédagogique est adaptable et l'enseignant peut modifier le "fil d'apprentissage » entre deux simulations lors du débriefing. Cependant, ce jeu présente trois limites importantes :

- Premièrement, le jeu étant basé sur des briques de LEGO, le lien entre les causes en situation (en usine) et celles de la réalité de la simulation demande un effort d'abstraction de haut niveau pour l'apprenant.

- Deuxièmement, la simulation ne prend pas en compte certains phénomènes liés au hasard dans un système de production : aucune panne n'est reproductible puisque c'est l'apprenant qui simule la machine et que son but est d'atteindre les objectifs de production quoiqu'il arrive.

- Troisièmement, les coûts sont difficiles à prendre en compte dans ce jeu. 


\subsection{Conception de Lea(r)nIT}

La conception de Lea(r)nIT repose sur l'instrumentation et l'informatisation du jeu décrit précédemment. L'architecture utilisée respecte l'architecture en trois niveaux proposée par Delomier et al. (2012) : interfaces utilisateurs, comportement de jeu et moteur de jeu.

Les interfaces utilisateur ont été conçues de façon à intégrer les environnements numériques et physiques sur une grande variété de supports et d'interactions. En ce sens, nous avons défini chaque poste comme suit :

1) Le poste de presse est représenté sur une table interactive avec interactions tactiles (équivalent aux boutons poussoirs sur une presse réelle).

2) Le poste d'assemblage est représenté sur une table interactive supportant des interactions tangibles. L'outil physique (un pistolet à colle augmenté d'une led infrarouge) est utilisé pour agir sur des données numériques (assembler et coller les différents composants du produit manufacturé). Le geste métier de ce poste de travail est donc reproduit comme sur un vrai poste d'assemblage.

3) La finalisation des produits est effectuée sur une table interactive supportant également les interactions tangibles. L'utilisation d'un potentiomètre et d'un "élément » de pointage physiques permet de reproduire les gestes spécifiques à l'ajustement de l'intensité et l'orientation du traitement appliqué sur le produit.

4) L'entrepôt est représenté sur une table Microsoft Pixel Sense. Différents espaces de travail sont représentés et le tracking d'objets permet de supporter différents gestes métiers tels que le réapprovisionnement de matières premières.

5) Le chariot du manutentionnaire est quant à lui représenté sur une tablette Android permettant de reproduire la mobilité et versatilité des situations entre deux postes de travail. Ainsi, lorsque le manutentionnaire se positionne sur le bord d'un poste de travail ou à l'entrepôt, il peut déposer ou prendre des produits.

6) Le contrôle qualité s'effectue sur un iPod Touch, en interaction avec la table Pixel Sense.

7) Enfin, une application contrôle (sur PC) permet aux expérimentateurs de contrôler l'ensemble du système et de fournir un certain nombre d'indicateurs.

Quatre illustrations des dispositifs sont présentées par les figures 3 et 4 page suivante. 
Sébastien GEORGE, Christine MICHEL, Audrey SERNA, Luca BISOGNIN
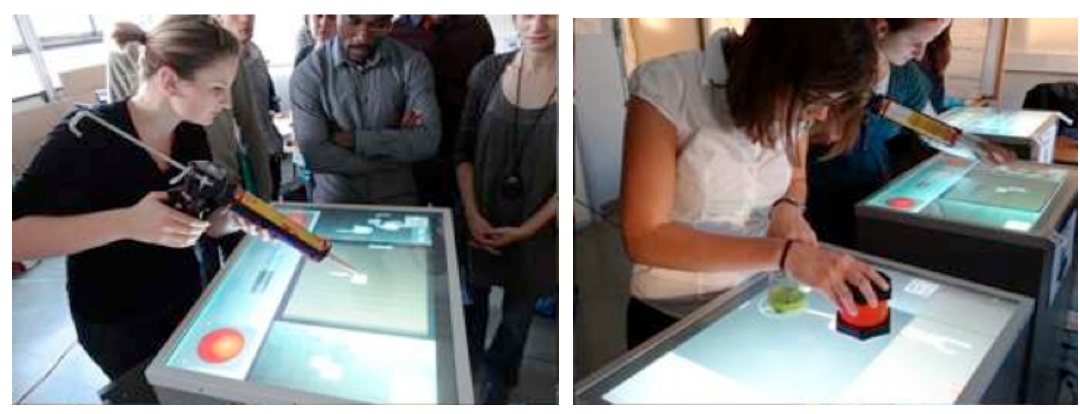

Figure 3 • Poste de travail d'assemblage et de spécification
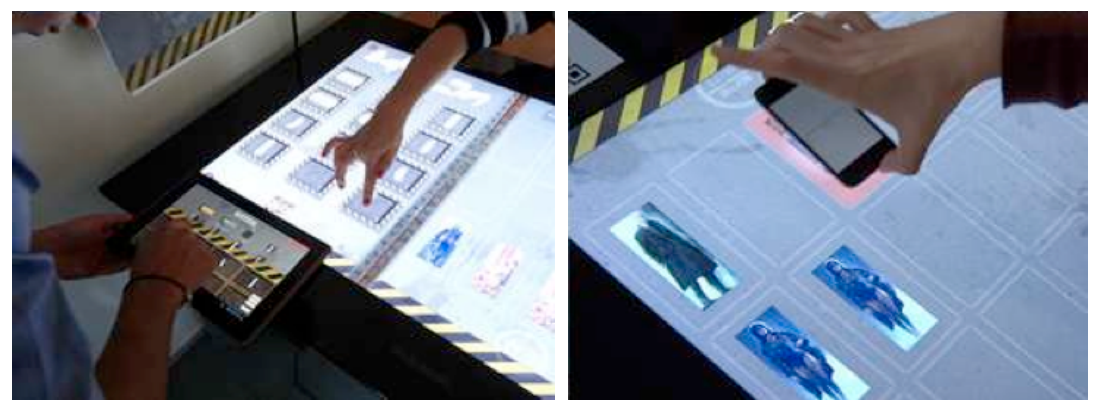

Figure 4 • Connexion entre le poste de contrôle qualité et les différents dispositifs mobiles

Ces choix de conception permettent d'évaluer différents niveaux d'immersion dans le jeu grâce aux interactions « numériques » (i.e. interactions directes sur des objets numériques) et tangibles (i.e. interactions sur des objets numériques via des objets physiques). Par exemple, le fait d'introduire dans la version instrumentée du jeu des gestes physiques permet de rendre compte de l'effort ou de la fatigue que peuvent ressentir les opérateurs sur certains postes de travail sur une ligne de production.

Concernant, la conception du moteur de jeu, l'un des enjeux principaux était la modélisation d'un monde persistent pour assurer la cohérence et l'intégrité des données du système dans son ensemble, tous supports confondus. Le modèle informatique adopté est décrit dans Delomier et al. (2012).

La transposition informatique du jeu permet de plus de faciliter le contrôle du système non seulement dans son ensemble mais également en finesse pour chaque sous-partie. En effet, différents indicateurs, comme le nombre de pièces produites ou les temps de production, sont fournis 
après chaque simulation induisant une compréhension plus réaliste des tâches et des situations. De plus, cette instrumentation nous donne l'opportunité d'introduire des évènements plus réalistes, tels que les pannes ou les produits avec des défauts qualité. De plus, nous pouvons prendre en compte les coûts des améliorations mises en place à chaque étape de la simulation.

\section{4. Étude expérimentale et résultats}

\subsection{Description du protocole expérimental}

De façon à évaluer les effets potentiels que peuvent avoir les LG en réalité mixte sur l'apprentissage, nous avons comparé deux cas de figure : un groupe de participants a utilisé le jeu original avec les briques de LEGO et des tables classiques pour simuler les postes de travail, un autre groupe de participant a utilisé le jeu Lea(r)nIT décrit précédemment. Le premier groupe est appelé groupe contrôle et correspond à la « condition LEGO ». Le second groupe correspond à la « condition Lea(r)nIT».

Chaque groupe était constitué de 7 participants recrutés parmi des étudiants volontaires du département Génie Industriel de l'INSA de Lyon et des doctorants du laboratoire LIRIS. Pour la première condition, le groupe était constitué de 6 hommes et 1 femme âgés entre 22 et 26 ans. Le deuxième groupe était constitué de 2 hommes et 5 femmes, eux aussi âgés entre 22 et 26 ans. Nous tenons à préciser dès maintenant que nous n'avons pas pris en compte le genre dans notre étude étant donnée la faible taille des échantillons. Une étude à plus grande échelle serait nécessaire pour tirer des conclusions à ce niveau. L'ensemble des participants des deux conditions, n'avait pas de connaissances approfondies sur la démarche Lean. Une formation d'une heure a donc été réalisée une semaine avant la session expérimentale pour leur donner les connaissances de base nécessaires à la réalisation du jeu.

Pour chaque condition, la session d'expérimentation s'est déroulée en 4 heures avec trois étapes distinctes : 1) courte présentation du projet de recherche et passation d'un pré-test permettant d'évaluer l'état des connaissances des participants sur les principes du Lean; 2) la session du jeu à proprement parler et 3) passation d'un post-test pour évaluer l'état des connaissances sur le Lean et recueil de l'expérience utilisateur.

Pour le cœur de l'expérimentation (la deuxième étape), nous avons fait appel à un enseignant de la démarche Lean au département Génie Industriel de l'INSA de Lyon. La session de jeu a commencé par une présentation de la ligne de production, des différents postes de travail et des sept 


\section{Sébastien GEORGE, Christine MICHEL, Audrey SERNA, Luca BISOGNIN}

différents rôles que les participants pouvaient prendre. Une première simulation s'est ensuite déroulée pendant 20 minutes et a été suivie d'un débriefing au cours duquel les participants ont analysé les gaspillages, les performances et les flux de production. Ils ont ensuite choisi des améliorations dans une liste d'améliorations possibles. Ces améliorations ont ensuite été déployées sur les postes de travail concernés et une deuxième simulation a été lancée pendant encore 20 minutes. Le débriefing suivant a permis aux participants d'observer et analyser les effets des améliorations choisies.

Comme mentionné précédemment, l'évaluation de l'expérimentation a été réalisée à l'aide de deux questionnaires. Le premier, appelé questionnaire pré ou post test, a été utilisé avant la session de jeu puis juste après pour pouvoir mesurer l'évolution des connaissances des sujets sur les concepts du Lean. Il a été conçu pour répondre à l'hypothèse $\mathrm{H} 1$, mais également plus finement aux hypothèses $\mathrm{H} 2$ et $\mathrm{H} 3$, avec des questions relatives à la compréhension des concepts théoriques ou pratiques. Pour $\mathrm{H} 2$, les apprentissages que l'on veut mesurer sont ceux qui portent sur la philosophie Lean de façon générale. Il est question entre autre de notion de valeur, concept central dans la démarche Lean, des deux piliers fondateurs de la démarche, à savoir le juste-à-temps (fournir le bon service ou produit au bon moment et en bonne quantité) et la qualité (fournir bon du premier coup), ou encore sur les caractéristiques des standards, les différents types de gaspillages, etc. Les concepts pratiques développés dans le scénario pédagogique et sur lesquels portent les questions d'évaluation correspondent à des améliorations possibles, comme l'application de la méthode des $5 \mathrm{~S}$ pour ranger et optimiser un poste de travail, ou encore savoir mesurer et améliorer le temps de traversée du produit dans l'usine (Lead Time) impactant sur les délais de livraison du client.

Le second questionnaire a été utilisé pour mesurer l'expérience utilisateur, l'utilisabilité du jeu en réalité mixte et la perception des participants concernant leur apprentissage.

\subsection{Résultats}

\subsection{1. Évaluation de I'expérience utilisateur avec Lea(r)nIT}

Comme nous venons de le mentionner, l'expérience utilisateur a été évaluée à partir d'un questionnaire qui a été proposé aux élèves après l'utilisation de Lea(r)nIT. Il était composé de questions fermées dont les réponses étaient données selon une échelle à quatre niveaux $(0=$ pas $d u$ tout d'accord, 1 = plutôt pas d'accord ; 2 = plutôt d'accord, 3 = complè- 
tement d'accord). Le tableau 1 montre que les étudiants qui ont utilisé Lea(r)nIT ont des avis plutôt positifs à propos de l'utilité, de la pertinence, du caractère ludique et de la facilité d'utilisation (avec des résultats moyens respectivement de 2,6,2,6,2,6 et 2,3). Le point de vue des étudiants est relativement homogène (l'écart type maximum est en effet à $0,7)$.

Tableau 1 - Avis des étudiants à propos de la conception générale de Lea(r)nIT

\begin{tabular}{|c|c|c|c|c|}
\hline Je pense que Lea(r)nIT est : & Moyenne & Écart type & Min & Max \\
\hline Utile & 2,6 & 0,5 & 2,0 & 3,0 \\
\hline Pertinent & 2,6 & 0,5 & 2,0 & 3,0 \\
\hline Drôle & 2,6 & 0,7 & 1,0 & 3,0 \\
\hline Facile à utiliser & 2,3 & 0,7 & 1,0 & 3,0 \\
\hline
\end{tabular}

Nous avons demandé aux participants leur niveau d'intérêt et leur sentiment de réussite dans la réalisation des étapes de simulation et d'amélioration. Le tableau 2 montre que les étudiants ont éprouvé de l'intérêt vis à vis de Lea(r)nIT à la fois pour les étapes de simulation et d'amélioration (avec des valeurs moyennes respectivement de $\mathrm{m}=2,6$ et $m=2,7)$. De plus ils se sont sentis capable de réaliser les deux activités (avec des valeurs moyennes respectivement de $\mathrm{m}=2,4$ et $\mathrm{m}=2,6$ ). On peut noter que les étudiants ont le sentiment d'avoir mieux réussi à réaliser les étapes d'amélioration que les étapes de simulation.

Tableau 2 • Intérêt et réussite à l'usage

\begin{tabular}{|c|c|c|c|c|}
\hline Quand j'utilise Lea(r)nIT: & Moyenne & Écart type & Min & Max \\
\hline $\begin{array}{c}\text { Je suis intéressé par } \\
\text { les phases de simulation }\end{array}$ & 2,6 & 0,5 & 2,0 & 3,0 \\
\hline $\begin{array}{c}\text { Je suis intéressé par } \\
\text { les phases d'amélioration }\end{array}$ & 2,7 & 0,5 & 2,0 & 3,0 \\
\hline $\begin{array}{c}\text { J'ai réussi à faire } \\
\text { les phases de simulation }\end{array}$ & 2,4 & 0,5 & 2,0 & 3,0 \\
\hline $\begin{array}{c}\text { J'ai réussi à faire } \\
\text { les phases d'amélioration }\end{array}$ & 2,6 & 0,5 & 2,0 & 3,0 \\
\hline
\end{tabular}

Nous avons ensuite demandé aux participants le niveau de ressenti de différentes émotions lors de l'utilisation de Lea(r)nIT. Les tableaux 3 et 4 montrent les réponses moyennes exprimées. Les émotions les plus souvent exprimées (tableau 3) sont positives comme le plaisir $(\mathrm{m}=2,4)$, 
Sébastien GEORGE, Christine MICHEL, Audrey SERNA, Luca BISOGNIN

l'excitation $(m=2,1)$ et la joie $(m=1,9)$. Quelques étudiants ont exprimés un sentiment d'euphorie $(\mathrm{m}=1,4)$ ou de surprise $(\mathrm{m}=1,4)$. La plupart des étudiants n'ont pas exprimé d'émotions négatives. Quelques étudiants ont exprimé un sentiment de stress (tableau 4) à l'idée de faire des erreurs de production $(m=1,6)$ ou des erreurs d'usage de la station de travail $(\mathrm{m}=1,1)$. Quelques étudiants ont aussi exprimé l'impression qu'ils n'arriveraient pas à suivre le rythme de production imposé par la chaîne lors de la simulation $(\mathrm{m}=1,3)$.

Tableau 3 • Émotions ressenties par les participants

\begin{tabular}{|c|c|c|c|c|}
\hline $\begin{array}{c}\text { Quand j'utilise Lea(r)nIT, } \\
\text { je ressens : }\end{array}$ & Moyenne & Écart type & Min & Max \\
\hline Du plaisir & 2,4 & 0,5 & 2,0 & 3,0 \\
\hline De l'excitation & 2,1 & 1,0 & 0,0 & 3,0 \\
\hline De la joie & 1,9 & 1,0 & 0,0 & 3,0 \\
\hline De l'euphorie & 1,4 & 0,7 & 0,0 & 2,0 \\
\hline De la surprise & 1,4 & 0,5 & 1,0 & 2,0 \\
\hline De la perplexité & 0,6 & 0,7 & 0,0 & 2,0 \\
\hline De l'embarras & 0,4 & 0,7 & 0,0 & 2,0 \\
\hline De la déception & 0,3 & 0,7 & 0,0 & 2,0 \\
\hline De la peur & 0,1 & 0,3 & 0,0 & 1,0 \\
\hline De la tristesse & 0,0 & 0,0 & 0,0 & 0,0 \\
\hline Du dégout & 0,0 & 0,0 & 0,0 & 0,0 \\
\hline De l'anxiété & 0,0 & 0,0 & 0,0 & 0,0 \\
\hline Du mépris & 0,0 & 0,0 & 0,0 & 0,0 \\
\hline
\end{tabular}

Tableau 4 • Stress ressenti par les participants

\begin{tabular}{|c|c|c|c|c|}
\hline $\begin{array}{c}\text { Quand j'utilise Lea(r)nIT, je } \\
\text { suis stressé à l'idée de : }\end{array}$ & Moyenne & Écart type & Min & Max \\
\hline $\begin{array}{c}\text { Faire des erreurs de produc- } \\
\text { tion }\end{array}$ & 1,6 & 0,5 & 1,0 & 2,0 \\
\hline $\begin{array}{c}\text { Faire des erreurs d'utilisation } \\
\text { de la station de travail }\end{array}$ & 1,1 & 0,8 & 0,0 & 2,0 \\
\hline $\begin{array}{c}\text { Ne pas réussir à suivre le } \\
\text { rythme de la production }\end{array}$ & 1,3 & 0,9 & 0,0 & 2,0 \\
\hline
\end{tabular}

\subsection{2. Évaluation des apprentissages}

\subsubsection{Sentiment d'apprentissage}


Les sentiments d'apprentissage exprimés par les participants sont présentés dans le tableau 5. Ils ont le sentiment d'avoir compris les concepts généraux du Lean $(\mathrm{m}=2,3$ pour le groupe LEGO et $\mathrm{m}=2,7$ pour le groupe Lea(r)nIT) et se sentent capable de mettre en application ces concepts dans des cas concrets $(\mathrm{m}=2,1$ pour le groupe LEGO et $\mathrm{m}=2,4$ pour le groupe $L e a(r) n I T)$. Les valeurs sont très comparables dans les deux groupes. D'ailleurs, si on fait un t-test (tableau 6) les écarts de valeurs concernant le sentiment d'apprentissage et la capacité de mise en œuvre ne sont pas significatifs (avec respectivement des valeurs $p=0,19$ et $p=0,4$ ).

On peut donc conclure que la perception d'apprentissage est similaire dans les deux groupes.

Tableau 5 • Sentiments d'apprentissage des participants

\begin{tabular}{|c|c|c|c|c|c|}
\cline { 3 - 6 } \multicolumn{2}{c|}{} & Moyenne & Ecart type & Min & Max \\
\hline $\begin{array}{c}\text { Condition } \\
\text { LEGO }\end{array}$ & $\begin{array}{c}\text { Je comprends les concepts } \\
\text { généraux du Lean }\end{array}$ & 2,3 & 0,5 & 2,0 & 3,0 \\
\cline { 2 - 6 } & $\begin{array}{c}\text { Je suis capable } \\
\text { d'appliquer les concepts } \\
\text { du Lean dans des cas con- } \\
\text { crets }\end{array}$ & 2,1 & 0,6 & 1,0 & 3,0 \\
\hline $\begin{array}{c}\text { Condition } \\
\text { Lea(r)nIT }\end{array}$ & $\begin{array}{c}\text { Je comprends les concepts } \\
\text { généraux du Lean }\end{array}$ & 2,7 & 0,5 & 2,0 & 3,0 \\
\cline { 2 - 6 } & $\begin{array}{c}\text { Je suis capable } \\
\text { d'appliquer les concepts } \\
\text { du Lean dans des cas con- } \\
\text { crets }\end{array}$ & 2,4 & 0,5 & 2,0 & 3,0 \\
\hline
\end{tabular}

Tableau 6 • Différence dans les sentiments d'apprentissage des participants

\begin{tabular}{|c|c|c|c|}
\hline t-test & $\mathrm{t}$ & $\mathrm{p}$-value & \\
\hline $\begin{array}{c}\text { Sentiment de compréhension des } \\
\text { concepts }\end{array}$ & $-1,367$ & 0,199 & Non significatif \\
\hline $\begin{array}{c}\text { Sentiment de capacité de mise en } \\
\text { œuvre des concepts }\end{array}$ & 0,866 & 0,403 & Non significatif \\
\hline
\end{tabular}

\subsubsection{Niveau de connaissance des participants avant les formations}

Le tableau 7 montre le niveau de connaissances des élèves, avant les formations, concernant les concepts du Lean. Les résultats sont regroupés selon que les questions qui ont été posées concernent des éléments théoriques ou pratiques du Lean. On peut noter que les participants du groupe $L e a(r) n I T$ ont des résultats généraux légèrement supérieurs que ceux du 
Sébastien GEORGE, Christine MICHEL, Audrey SERNA, Luca BISOGNIN

groupe LEGO ( $\mathrm{m}=13,4$ pour le groupe LEGO et $\mathrm{m}=14,9$ pour Lea(r)nIT). Plus spécifiquement, le groupe Lea(r)nIT a de meilleurs résultats sur les questions théoriques $(\mathrm{m}=8,4$ pour le groupe LEGO $v s \mathrm{~m}=10,8$ pour le groupe Lea(r)nIT) alors que le groupe LEGO a de meilleurs résultats sur les question pratiques $(\mathrm{m}=5$ pour le groupe LEGO $v s \mathrm{~m}=4$ pour le groupe Lea(r)nIT). On peut remarquer que globalement les deux groupes ont de meilleurs résultats sur les questions théoriques que sur les questions pratiques.

Tableau 7 • Résultats au test de connaissances avant l'expérimentation

\begin{tabular}{|c|c|c|c|c|c|}
\hline & Avant l'expérimentation & Moyenne & Écart type & Min & Max \\
\hline $\begin{array}{c}\text { Condition } \\
\text { LEGO }\end{array}$ & $\begin{array}{c}\text { Ensemble des connais- } \\
\text { sances }\end{array}$ & 13,4 & 5,1 & 6,2 & 23,7 \\
\cline { 2 - 6 } & Connaissances théoriques & 8,4 & 3,1 & 5 & 14,3 \\
\cline { 2 - 6 } & Connaissances pratiques & 5 & 3 & 0 & 9,5 \\
\hline $\begin{array}{c}\text { Condition } \\
\text { Lea(r)nIT }\end{array}$ & $\begin{array}{c}\text { Ensemble des connais- } \\
\text { sances }\end{array}$ & 14,9 & 5 & 9,5 & 23,5 \\
\cline { 2 - 6 } & Connaissances théoriques & 10,8 & 3 & 7 & 15,5 \\
\cline { 2 - 6 } & Connaissances pratiques & 4 & 2 & 0,5 & 8 \\
\hline
\end{tabular}

Nous avons analysé si les différences entre les deux groupes étaient significatives en utilisant un t-test. Les résultats présentés dans le tableau 8 montrent qu'il n'y a pas de différence significative sur l'état des connaissances initiales à propos du Lean entre les deux groupes à la fois sur le plan général $(p=0,634)$ et sur les connaissances théoriques $(p=0,201)$ ou pratiques $(p=0,540)$. Nous pouvons donc en conclure que le niveau de connaissances initiales relatif au Lean des participants est équivalent avant l'expérimentation pour les deux groupes.

Tableau 8 - Différence entre les groupes dans les connaissances en Lean avant l'expérimentation

\begin{tabular}{|c|c|c|c|}
\hline t-test avant l'expérimentation & $\mathrm{t}$ & $\mathrm{p}$-value & \\
\hline Ensemble des connaissances & $-0,489$ & 0,634 & Non significatif \\
\hline Connaissances théoriques & $-1,353$ & 0,201 & Non significatif \\
\hline Connaissances pratiques & 0,631 & 0,540 & Non significatif \\
\hline
\end{tabular}

4.2.2.3. Niveau de connaissance des participants après les formations

Le tableau 9 montre le niveau de connaissances des élèves, après les formations. On peut voir que le groupe Lea(r)nIT a de meilleurs résultats 
sur l'ensemble des compétences que le groupe LEGO $(\mathrm{m}=15,9$ pour le groupe LEGO $v s \mathrm{~m}=21,9$ pour le groupe Lea(r)nIT). Le groupe Lea(r)nIT a en particulier de meilleurs résultats concernant les questions théoriques $(\mathrm{m}=8,5$ pour le groupe LEGO $v s \mathrm{~m}=13,3$ pour le groupe Lea(r)nIT). Les résultats sont aussi meilleurs concernant les questions pratiques mais de manière bien moins marquée $(\mathrm{m}=7,4$ pour le groupe LEGO $v s \mathrm{~m}=7,6$ pour le groupe $L e a(r) n I T$ ). Les deux groupes ont de meilleurs résultats sur les questions théoriques que sur les questions pratiques.

Tableau 9 • Résultats au test de connaissances après l'expérimentation

\begin{tabular}{|c|c|c|c|c|c|}
\hline & Après l'expérimentation & Moyenne & Écart type & Min & Max \\
\hline \multirow{2}{*}{$\begin{array}{c}\text { LEGO } \\
\text { condition }\end{array}$} & $\begin{array}{c}\text { Ensemble des connais- } \\
\text { sances }\end{array}$ & 15,9 & 4,3 & 9,5 & 22,25 \\
\cline { 2 - 6 } & Connaissances théoriques & 8,5 & 2,4 & 5 & 12 \\
\cline { 2 - 6 } & Connaissances pratiques & 7,4 & 2,4 & 3,5 & 11,5 \\
\hline \multirow{2}{*}{$\begin{array}{c}\text { Lea(r)nIT } \\
\text { condition }\end{array}$} & $\begin{array}{c}\text { Ensemble des connais- } \\
\text { sances }\end{array}$ & 21,9 & 4,1 & 15,8 & 28,3 \\
\cline { 2 - 6 } & Connaissances théoriques & 13,3 & 2,2 & 11,3 & 18,3 \\
\cline { 2 - 6 } & Connaissances pratiques & 7,6 & 2,8 & 2,8 & 10,8 \\
\hline
\end{tabular}

Les résultats du tableau 10 montrent que, selon un t-test, les différences entre les deux groupes sont significatives si on considère l'ensemble des connaissances $(p=0,059)$ et les connaissances théoriques $(\mathrm{p}=0,003)$ alors $q \mathrm{u}^{\prime}$ elles ne le sont pas si on considère les connaissances pratiques $(\mathrm{p}=0,869)$.

Tableau 10 - Différence entre les groupes dans les connaissances en Lean après l'expérimentation

\begin{tabular}{|c|c|c|c|}
\hline $\mathrm{t}$-test après l'expérimentation & $\mathrm{t}$ & $\mathrm{p}$-value & \\
\hline Ensemble des connaissances & $-2,081$ & 0,059 & Significatif \\
\hline Connaissances théoriques & $-3,626$ & 0,003 & Significatif \\
\hline Connaissances pratiques & $-0,168$ & 0,869 & Non significatif \\
\hline
\end{tabular}

Nous pouvons en conclure que les participants du groupe Lea(r)nIT ont globalement plus appris que les étudiants du groupe LEGO. Donc l'hypothèse $\mathrm{H} 1$ est validée. De plus, les participants qui ont utilisé Lea(r)nIT ont au final une meilleure compréhension des concepts théoriques que les étudiants du groupe LEGO. Donc l'hypothèse H2 est validée. Cependant nous n'avons pas pu montrer que l'utilisation de 


\section{Sébastien GEORGE, Christine MICHEL, Audrey SERNA, Luca BISOGNIN}

Lea(r)nIT procurait une meilleure construction de compétences pratiques. L'hypothèse H3 n'est donc pas validée et nous dégageons des raisons possibles dans la partie suivante.

\subsection{Discussion}

Les résultats généraux à propos de l'utilisabilité et de l'expérience utilisateur sont très positifs. Les étudiants pensent que la conception générale de Lea(r)nIT est bonne. Ils pensent en particulier que Lea(r)nIT est utile, pertinent, amusant et facile à utiliser. Ils se déclarent intéressés à utiliser Lea(r)nIT à la fois pour réaliser les étapes de simulation et d'amélioration de l'activité proposée. Ils ont le sentiment de bien réussir à réaliser ces deux étapes en l'utilisant. Ils expriment des émotions très positives comme le plaisir, l'excitation, la joie et ne ressentent pas d'émotions négatives comme l'embarras, la déception, la peur, la tristesse, le dégout ou la colère. Ils n'expriment pas non plus de sentiment de stress lié au rythme de l'activité ou aux erreurs que la manipulation des dispositifs de RM induirait. Ainsi, nous pouvons conclure que la conception de Lea(r)nIT n'engendre pas de problème d'utilisabilité.

Sur le plan des apprentissages, les premiers résultats présentés ici sont encourageants. En effet, notre étude montre que l'apprentissage est meilleur si les outils et objets manipulés lors de la formation sont réalisés en RM plutôt que de façon plus symbolique comme c'est le cas avec les LEGO. La justification de ce résultat est liée selon nous au fait que le degré de réalisme et l'immersion rendus possible par la RM permettent aux participants de faire plus simplement le lien entre les éléments avec lesquels ils interagissent dans le jeu et la réalité de l'activité qui est simulée. En effet, le paradigme de l'apprentissage par l'action suppose qu'en plaçant l'apprenant dans une situation où les gestes et l'interaction sont plus réalistes, l'action située sera mieux définie et l'expérience vécue plus propice aux apprentissages.

Nous nous interrogions sur les possibles différences d'impacts sur l'apprentissage des concepts théoriques et pratiques. En effet, si l'environnement et les actions sont plus réalistes, on peut s'attendre, d'une part, à ce que les participants aient une meilleure prise de recul sur leurs activités ainsi qu'une meilleure compréhension des enjeux et méthodes de la situation qui est représentée (concepts théoriques). On peut s'attendre, d'autre part, à ce que la maîtrise des gestes et attitudes soit plus grande (concepts pratiques). Notre étude montre que les concepts théoriques du domaine sont mieux compris s'il y a usage de la RM dans le Se- 
rious Game, en revanche aucune amélioration significative n'a été observée sur les concepts pratiques.

Nous pensons que ces résultats sont liés aux objectifs pédagogiques de la formation et au scénario du jeu initial sur lequel nous nous sommes appuyé. En effet, les aspects pratiques du Lean qui sont vus dans la formation consistent à comprendre comment mettre en ouvre des méthodologies (comme les 5S) et comment contrôler le déroulement de la production (par des activités de type mesure du temps de transfert d'une pièce par exemple). Les aspects théoriques consistent à comprendre la philosophie de la méthode Lean, les différents éléments de la production sur lesquels il est intéressant d'agir et comment ces changements améliorent le processus industriel à plus grande échelle. Le scénario du jeu et le fait de jouer des rôles aident surtout les participants à se mettre à la place des opérateurs, comprendre quel est leur travail et les bénéfices ou contraintes liés au déploiement d'une démarche Lean. Les gestes réalisés sur les plateformes de travail, en utilisant des techniques de RM, ne visent donc pas l'acquisition de certaines compétences pratiques liées aux objectifs pédagogiques. Le seul rôle qui expérimente une compétence pratique est l'observateur qui mesure les temps de production à l'aide d'un chronomètre au cours de l'activité. Ses mesures servent principalement à alimenter les discussions lors des phases de débriefing avec l'enseignant. C'est en effet à ce moment que les compétences pratiques de la formation sont discutées et approfondies. Le travail de l'observateur et les débriefings étant réalisés de manière équivalente dans les deux cas, l'absence de différences d'apprentissage sur les concepts pratiques est justifiée. En revanche, notre expérimentation montre bien que l'ajout d'interaction en RM lors des phases de simulation aide les participants à mieux comprendre les concepts théoriques liés au Lean. En effet, les concepts théoriques sont présentés rapidement à l'oral pour les deux groupes en début de formation, expérimentés de manière différenciée lors des phases de simulation et discutés eux aussi, de manière équivalente pour les deux groupes lors des phases de débriefing. Nous pouvons donc bien conclure que c'est l'ajout des techniques de RM qui améliore la compréhension générale de la méthode Lean et de la manière de la mettre en œuvre.

Pour améliorer l'apprentissage sur les concepts pratiques, nous pensons qu'il serait utile de développer des interactions utilisant de la RM lors des phases d'amélioration pour soutenir l'étape de débriefing et de discussion sur l'intérêt de proposer telle ou telle amélioration. On peut imaginer par exemple qu'à l'issue des simulations, les élèves manipulent des arte- 


\section{Sébastien GEORGE, Christine MICHEL, Audrey SERNA, Luca BISOGNIN}

facts représentatifs des méthodes d'observation et de contrôle du Lean, proposent des stratégies d'amélioration, réorganisent la ligne de production et visualisent sur le poste de contrôle, par le biais d'une simulation à horizon d'une journée ou d'une semaine, les impacts sur la production. Les élèves pourraient faire ce travail dans un premier temps individuellement puis discuter les différents choix en groupe sur la base des effets qu'ils produisent. On peut imaginer aussi que ces choix soient testés et discutés en groupe en utilisant une table multi-touch et des interfaces tangibles et qu'une discussion globale soit ensuite faite sur la base de la solution proposée collaborativement. Ces activités auraient pour conséquence d'approfondir les discussions et ainsi la compréhension des méthodes de déploiement du Lean. Néanmoins, le fait de pouvoir voir les effets des choix sur des simulations en accéléré aurait pour conséquence de limiter l'intérêt de les vivre en contexte simulé avec de la RM. Pour garder le scénario global du jeu attractif, en particulier le fait de jouer les phases de simulation, il faudrait ajouter des éléments de jeu lors des phases d'amélioration et laisser à l'enseignant la possibilité de définir les éléments les plus adaptés selon les propositions des élèves. Cette limite a par exemple été soulignée par l'enseignante qui a réalisé la formation. Cette personnalisation, qui impliquerait une modification du scénario implémenté, ne faisait pas partie des objectifs fixés dans cette recherche. Nous sommes cependant intéressés à la prendre en compte dans une prochaine itération à présent que les premières observations ont été faites.

Ces résultats doivent être considéré cependant avec prudence car la taille de l'échantillon de participants était relativement petite (14 participants au total). Ceci est lié aux contraintes expérimentales et aussi au jeu initial que nous avons choisi de transposer. En effet, les sept rôles qui sont définis dans le scénario contraignent le nombre de participants par séance. De plus, nous avons demandé à la même personne de faire l'animation de la formation dans les deux cas pour limiter les biais liés à la manière de travailler de l'enseignant. Dans la mesure où cette formation dure quatre heures pour chaque groupe, il a été impossible pour cette personne d'encadrer plus de groupes.

\section{Conclusion}

Nous avons constaté qu'il y avait encore peu de travaux autour des MRLG (Mixed Reality Learning Games) et que leurs effets sur l'apprentissage n'étaient pas réellement mesurés. Le projet SEGAREM a pour objectif d'étudier le processus de conception ainsi que l'utilité de ces nouvelles formes d'interaction sur l'apprentissage. 
Dans le cadre de ce projet, nous avons conçu un MRLG appelé Lea(r)nIT. De manière à tester les effets de la RM, nous avons choisi d'adapter un serious game non numérique déjà existant, le Buckingham Lean Game. Ce jeu est notamment utilisé depuis plusieurs années dans une école d'ingénieurs pour former les étudiants à la démarche Lean. Le jeu consiste à faire tenir aux élèves différents rôles dans une chaîne de production (phase de simulation) de manière à les aider à appréhender les gaspillages et perte de temps. Dans un deuxième temps, les étudiants sont amener à réfléchir aux différentes méthodes du Lean puis les mettent en application (phase de débriefing et amélioration). Les rôles correspondent à différents opérateurs d'une chaîne de production (presse, assemblage, contrôle qualité...). De manière à simuler la production de pièces, les élèves travaillent avec des boites en plastiques (pour représenter les supports de stockage) et des blocs de LEGO (pour représenter les pièces), qu'ils s'échangent et qu'ils assemblent en suivant un processus de production fourni. Notre travail de conception a consisté à remplacer l'utilisation des LEGO par des techniques de RM. Le scénario du jeu ainsi que les objectifs pédagogiques sont restés inchangés. Ainsi la version MRLG de la formation utilise différents artefacts : 4 tables interactives (3 ont été conçues spécifiquement dans le cadre du projet, la dernière est une table Pixel Sense), une tablette et un smartphone. En complément, des objets ont été utilisés comme interfaces tangibles de manière à accroître le sentiment d'immersion des apprenants dans l'activité. Par exemple, un pistolet à colle a été adapté de manière à pouvoir poser des points de colle sur des pièces virtuelles qui sont manipulées sur une table interactive représentant le poste d'assemblage.

Les deux situations ont ensuite été évaluées en utilisant une méthode expérimentale. 14 participants (étudiants d'école d'ingénieurs) ont été soumis à deux conditions de formation : 7 pour la situation LEGO et 7 pour la situation Lea(r)nIT. Les pré-tests et post-tests sur l'état des connaissances ont montré que l'utilisation de la RM impactait positivement l'apprentissage, en particulier concernant la compréhension des concepts théoriques. Les observations complémentaires montrent que ces résultats s'expliquent principalement par une plus grande motivation, une meilleure immersion et une meilleure expérience utilisateur. De plus, nous pensons que le fait de réaliser des activités plus réalistes aide les élèves à mieux se projeter dans la simulation et ainsi à comprendre de manière plus fine les dysfonctionnements qui justifient l'emploi de telle ou telle méthode ainsi que les conséquences que cela peut avoir sur la production. Les concepts théoriques de la formation sont ainsi compris avec plus de 


\section{Sébastien GEORGE, Christine MICHEL, Audrey SERNA, Luca BISOGNIN}

sens. Néanmoins, nous n'avons pas identifié de différences significatives sur les apprentissages concernant les concepts pratiques. Nous pensons que ce résultat est lié à l'organisation de la formation qui vise à former les étudiants à découvrir la méthode et avoir une vision globale de l'analyse de la situation et des améliorations possibles que l'on peu faire. Nous pensons que nous aurions pu démontrer l'avantage de l'utilisation de la RM sur la compréhension fine d'aspects pratiques en modifiant le scénario pédagogique lors des phases de débriefing, par exemple en utilisant une table ou des tablettes tactile pour définir collaborativement les améliorations possibles et en voir les effets à plus ou moins long terme. Mais ce choix aurait nécessité de repenser le scénario pédagogique dans sa globalité de manière à garder l'étape de simulation de la chaîne de production intéressante et conserver la découverte graduelle des améliorations.

Nos résultats vont dans le même sens que ceux de Cuendet et al. (2012) qui montrent que l'utilisation d'interfaces tangibles tend à modifier les comportements des apprenants, les encourage à manipuler plus mais aussi parfois à réfléchir moins, ce qui peut dans ce cas avoir un impact négatif sur les apprentissages. La réalité mixte en formation ne doit pas être juste considérée pour l'assistance qu'elle fournit pour la réalisation de tâches manuelles. Elle doit être aussi vue comme un moyen d'encourager une réflexion plus profonde chez les élèves. Dans cette perspective, le rôle des enseignants reste fondamental pour les aider à exploiter ces nouvelles expériences vécues, prendre du recul sur leurs activités, faire le lien avec les concepts présentés et ainsi mieux apprendre. Ce rôle peut se manifester aussi dans la mise en ouvre du dispositif par le choix, en cohérence avec le scénario général, des activités à réaliser selon les réactions et propositions des élèves. Cette possibilité d'adaptation nous semble particulièrement utile pour favoriser l'appropriation de ces dispositifs par les enseignants. Elle constitue une de nos prochaines perspectives de recherche.

\section{Remerciements}

Cette recherche a été réalisée dans le cadre du projet SEGAREM (SErious GAmes et REalité Mixte). Les auteurs tiennent à remercier à la fois la DGCIS (Direction Générale de la Compétitivité, de l'Industrie et des Services) qui a accepté de le financer, et les partenaires industriels, Symetrix

et Total Immersion, pour leur collaboration. Les auteurs tiennent aussi à 
exprimer leur gratitude aux étudiants de l'INSA de Lyon et à leur enseignante Lorraine Trilling, pour avoir accepté de participer à l'expérimentation.

\section{BIBLIOGRAPHIE}

ABT C. C. (1987). Serious games. University Press of America.

ANASTASSOVA M., BURKHARDT J.-M. (2008). Automotive technicians' training as a community-of-practice: implications for the design of an augmented reality teaching aid. Applied ergonomics, 40(4), 713-721.

CUENDET S., JERMANN P., DILLENBOURG P. (2012). Tangible interfaces: when physical-virtual coupling may be detrimental to learning. In Proceedings of the $26^{\text {th }}$ Annual BCS Interaction Specialist Group Conference on People and Computers, Swinton, UK, UK: British Computer Society, p. 49-58.

CIPE (2013), Centre International de la Pédagogie d'Entreprise, http://www.cipe.fr/jeux-et-seminaires.html

CRAWFORD C. (1982). The art of computer game design. Osborne/McGraw-Hill.

DAVID B., YIN, C., CHALON R. (2009). Contextual Mobile Learning Strongly Related to Industrial Activities: Principles and Case Study. International Journal of Advanced Corporate Learning, Vol. 2 (3), 12-20.

DELOMIER F., DAVID B., CHALON R. (2012). Conception et mise en œuvre de Learning Games Collaboratifs Contextualisés. In SYSCO, Première conférence francophone sur les systèmes Collaboratifs, 28-30 sept. 2012.

De FREITAS S., NEUMANN, T. (2009). The use of 'exploratory learning' for supporting immersive learning in virtual environments. Computers and Education Journal, 52(2), 343-352.

GARRIS R., AHLERS R., DRISKELL J. (2002). Games, motivation, and learning: A research and practice model. Simulation \& Gaming, 33(4), 441-467.

HERRINGTON J., OLIVER R. (2000). An instructional design framework for authentic learning environments. Educational Technology Research and Development, Vol. 48 (3), 23-48.

JOAB M., GOUARDÈRES G. (dir.) (2000). Simulation et formation professionnelle dans l'industrie. Sciences et Techniques Éducatives (STE) numéro spécial, Hermès-Lavoisier, 232 p.

JOURDE F., LAURILLAU Y., NIGAY L. (2010). COMM notation for specifying collaborative and multimodal interactive systems. Proceedings of the $2^{\text {nd }} A C M$ SIGCHI symposium on Engineering interactive computing systems, p.125-134.

KRIZ W.C. (2010). A Systemic-Constructivist Approach to the facilitation and Debriefing of Simulations and Games. Simulation and Gaming: An International Journal, 41, 663-680.

LAVE J., WENGER E. (1991). Situated Learning. Legitimate Peripheral Participation. Cambridge University Press.

MARFISI-SCHOTTMAN I., GEORGE S., TARPIN-BERNARD F. (2010). Tools and Methods for Efficiently Designing Serious Games. Proceedings of $4^{\text {th }}$ Europeen Conference on Games Based Learning, Copenhagen, Denmark, p. 226-234. 


\section{Sébastien GEORGE, Christine MICHEL, Audrey SERNA, Luca BISOGNIN}

MARIAIS C., MICHAU F., PERNIN J.-P., MANDRAN N. (2011). Supporting Learning Role-Play Games Design: A Methodology and Visual Formalism for Scenarios Description. Proceedings of the 5th European Conference on Games Based Learning, Athens, Greece, p. 378-387.

MILGRAM P., KISHINO F. (1994). A Taxonomy of Mixed Reality Visual Displays. IEICE Transactions on Information and Systems, E77-D(12), 1321-1329.

ORLIAC C., GEORGE S., MICHEL C., PRÉVOT P. (2011). Can we use Existing Pedagogical Specifications to Design Mixed Reality Learning Games? Proceedings of the 5th European Conference on Games Based Learning, Athens, Greece, p. 440-448.

ORLIAC C., MICHEL C., GEORGE S. (2012). An Authoring Tool to Assist the Design of Mixed Reality Learning Games. $7^{\text {th }}$ European Conference of Technology Enhanced Learning, Saarbrücken, Germany, September 2012, p. 441-446

PRINCE M. (2004) Does Active Learning Work? A Review of the Research. Journal of Engineering Education, Vol. 93, $\mathrm{n}^{\circ} 3,223-231$.

STEDMON A.W., STONE R.J. (2001). Re-viewing reality: human factors of synthetic training environments. International Journal of Human-Computer Studies, 55(4), 675-698.

SUCHMAN L. (1987). Plans and situated actions : the problem of human-machine communication, Cambridge University Press, New York, 1987.

SQUIRE K., JENKINS H. (2003). Harnessing the power of games in education. Insight, vol. $3, \mathrm{n}^{\circ} 1,5-33$. 